\title{
Article
}

\section{Exploring team working in dentistry using a process model of team effectiveness}

\author{
Willcocks, Stephen George \\ Available at http://clok.uclan.ac.uk/21299/ \\ Willcocks, Stephen George ORCID: 0000-0002-1764-5951 (2018) Exploring \\ team working in dentistry using a process model of team effectiveness. \\ British Journal of Healthcare Management . ISSN 1358-0574
}

It is advisable to refer to the publisher's version if you intend to cite from the work. 10.12968/bjhc.2018.24.2.77

For more information about UCLan's research in this area go to http://www.uclan.ac.uk/researchgroups/ and search for < name of research Group>.

For information about Research generally at UCLan please go to http://www.uclan.ac.uk/research/

All outputs in CLoK are protected by Intellectual Property Rights law, including Copyright law. Copyright, IPR and Moral Rights for the works on this site are retained by the individual authors and/or other copyright owners. Terms and conditions for use of this material are defined in the policies page.

\section{CLoK}

Central Lancashire online Knowledge www.clok.uclan.ac.uk

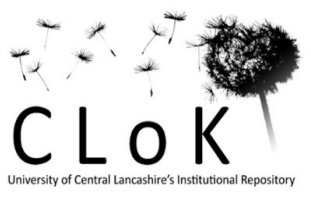




\section{Exploring team working in dentistry using a 'process' model of team effectiveness}

\section{Background}

NHS Dentistry is set against a background of policy change which includes the following objectives: improving oral healthcare, particularly quality, prevention, and reducing inequalities; improving access to dentistry; improving information for patients; developing a care pathway approach and integrated care; fostering patient and public engagement; and improving the workforce, (NHS England, a 2014). Other changes relate more specifically to the existing dental contract (2006) which currently gives more emphasis to treatment than prevention and highlights quantity as opposed to quality. NHS England, as commissioner of primary and secondary care dentistry and holder of dental contracts, wants to focus more on oral health improvement and good clinical outcomes, not just clinical activity, (NHS England, a 2014).A new proto -type dental contract is being piloted by a number of dental practices based on a philosophy of prevention and to be delivered by a care pathways approach incorporating a dental quality and outcomes framework, (Hatton, 2017).

Changes are also occurring to the structure of dentistry, which is currently a mixed economy of private and NHS dentistry, (GDC, 2013 a). Corporate dentistry is now a growing and influential part of this mixed economy providing alternative employment opportunities for dentists. This is likely to affect the future dental workforce. Wider changes include the possible impact of the UK leaving the EU. While this is currently being negotiated, this may have an impact on education, training, recruitment, and EU recognition for UK dentists, (Wilson, 2017).

This paper will explore team working in dentistry in this changing context using the inputprocess-output model of team effectiveness, (Salas, et al, 2008, Borrill, et al, 2000). Team working is seen as the optimal way of delivery by key organisations like the GDC (General Dental Council), (Morison, and McMullan, 2013, GDC 2013 b). Indeed, it is considered to be 'a key mantra of contemporary healthcare, (Bleakley, 2013), notwithstanding a lack of evidence on how it can be used and developed, (Ezziane, et al,2012).

\section{Definitions}

There are numerous definitions of 'teams' or 'team working', related to the fact that teams differ by virtue of the differing challenges they face,(Mathieu, et al, 2008). It is possible to describe teams according to differences in three core dimensions: skill differentiation; authority differentiation; and temporal stability, (West and Lyubovnikova, 2013, p135). In this view, teams differ in terms of whether they are uni-disciplinary or interdisciplinary; by the extent to which team members are involved in decision making; and whether the team is temporary or permanent. In the case of dentistry, teams are interdisciplinary- dentist, dental nurse, dental therapist / hygienist, dental technicians- but with the core role occupied by the dentist, and tend to be relatively permanent, depending on staff retention 
and turnover within a particular practice. In terms of decision making, dentistry has traditionally been carried out in an independent practice, (GDC, 2013 a), in which dentists have had a prerogative over decision-making as owner of the practice. One consequence is that this may have acted as a barrier to team- based decision making, particularly given that dentists employ other members of the team.

Alternatively, it is possible to define a 'team' in terms of the process of team working. Thus, a team is 'premised upon the organisation of people with a shared purpose and the way in which they work together to achieve this,' (Gopee and Galloway, 2009, p180). Similarly, 'team working' may be defined as 'the ability of a group of individuals to work together', (Ezziane, et al,2012). A more detailed definition suggests that team working is:

'a dynamic process involving two or more health professionals with complementary backgrounds and skills, sharing common health goals and exercising concerted physical and mental effort in assessing, planning, or evaluating patient care. This is accomplished through interdependent collaboration, open communication, and shared decision making,' (Xyrichis, and Ream, 2008).

Emphasis on the process of team working is said to be important where such teams work on complex, difficult tasks in stressful environments, and are required to undertake errorfree work, (Salas, et al, 2008, p540). This is the case in dentistry where teams generally rely on a collaborative process, share similar backgrounds and skills, plan and coordinate their work activities, and share common goals. In fact, collaborative working is the basis for delivering patient- focused care, (Reeson, et al, 2013 p5). This will be particularly relevant with the development of new forms of delivery such as managed clinical networks and implementation of the new dental contract with the care pathways approach, (Hatton, 2017).. The emphasis on process has been a feature of theory about teams and team working and has influenced the development of various models of team working, not least a 'process' model of team effectiveness.

\section{Theory}

The theory about how teams work has been dominated, in particular, by the 'input-processoutput' model, (Mathieu, et al, 2008; Borrill, et al,2000, Salas, et al, 2008 ). This 'process' model attempts to capture the key variables associated with team performance or effectiveness, ie the extent to which inputs and the process lead to the desired outputs, (summarised in fig 1).

\begin{tabular}{|l|l|l|}
\hline Inputs > & Process > & Outputs \\
\hline $\begin{array}{l}\text { Organisational context and } \\
\text { environment } \\
\text { Team characteristics }\end{array}$ & $\begin{array}{l}\text { Leadership and decision } \\
\text { making }\end{array}$ & $\begin{array}{l}\text { Patient outcomes/clinical } \\
\text { effectiveness }\end{array}$ \\
& & \\
\hline
\end{tabular}

Fig 1

Inputs 
Inputs derive from the context or wider environment, and, according to research evidence, the wider environment from which they are drawn is an important influence on team performance, (Borrill, et al,2000, p365). Key variables or inputs influencing performance include those related to team composition, such as personality, cognition, motivation, and culture; those related to work structure, such as norms, communication, structure, job assignments; and those related to task characteristics, such as workload, task type, and interdependency, (Salas, et al, 2008, p543).

With regard to team composition, individual attributes of team members, such as cognitive ability, drive, and enthusiasm, alongside professional training and competence, contribute to both the effectiveness of the team process, and the quality of outputs. Similarly, the personality- type of individual team members may influence their ability to work together as a cohesive team. The culture of dentistry is also relevant. This may be described as a hybrid of professional and 'business' culture, the result of the fact that dentists- in addition to providing a professional service- are independent contractors who work in SMEs (small / medium-sized business enterprises). The existence of a hybrid culture in dentistry, with potentially differing values and assumptions, may be a source of tension or conflict. For example, conflict may occur as a result of differing personal, financial or clinical objectives. Differences in culture and individual characteristics may act as a barrier to team working and collaboration, (Hall, 2005), and may result in poor communication, misunderstanding, role conflict, or personality clashes, with a negative effect on overall performance.

Similarly, inputs include the way in which work is structured and assigned in dental practice. These are influenced by the existing dental contract of 2006, and the pressures of the latter on funding and workload. (It has been noted that the contract is under review). Workload factors can be related to the possibility of work- based stress in dentistry, exacerbated by the increasing threat of litigation. A study has highlighted potential work stressors in dentistry:

'An important finding was that factors in the dental surgery explained nearly half of the overall stress in a GDP's life, especially fragility of dentist-patient relationship, time and scheduling pressures, staff and technical problems, job dissatisfaction, percentage NHS and number of hours worked per week', (Myers, and Myers, (2004).

Inputs relating to task characteristics- in particular, task- type and inter-dependency- are also relevant in relation to their possible impact on performance. In the case of dentistry, such inputs may be in the process of transition in order to accommodate changes to skill mix involving dentists and other members of the team, and the development of dentists with enhanced skills. Indeed, it is noted that the 'concept of the dentist leading a flexible workforce offering an interchangeable mix of skills has been around for many years', (Csikar, et al, 2009, p529). Depending on the task- type profile of the practice and its mission, there may be specific inputs where the practice decides to offer specialist services alongside general dentistry. Specialist inputs may also be provided if hospital dental specialties provide some services in the community as part of the development of integrated care. 
Inputs also include aspects of the wider environment in which teams work and the challenges that may be presented, for example, the challenges arising from the oral health status of the practice population, or the extent of competition between dental practices. With regard to the former, it is noted that levels of oral disease are highest in deprived areas, so there may be particular challenges for dentistry in some locations, (NHS England, 2014 b). The growth of corporate dental organisations may be a challenge in terms of competition with existing practices. Indeed, this may also have an impact on the culture in dentistry, with the possibility of reduced professional autonomy for dentists working in this context, (GDC, 2013 a).

\section{Process}

The above inputs contribute to the effectiveness of the team process. The latter is particularly important in dentistry, in fact, 'team processes' have a central role in most models of team effectiveness, (Mathieu, et al,2008). One of the most important influences on the team process is leadership and how this impacts on decision making within the practice, and on team members, in particular, their motivation and well-being, ( AlimoMetcalfe, and Bradley,2009). Leadership is also important in terms of quality. The CQC (Care Quality Commission) has noted in its inspection reports that being 'well-led' and having 'engaged and empowered staff' are fundamental to meeting its quality standards, (Harris, 2016). Leadership may be provided individually or shared within the team (or a combination of the two) In the case of dentistry this has been traditionally exercised by dentists/practice owners, who have formal responsibilities. However, a large scale study of leadership in the NHS reported that the 'heroic' individual leader is being replaced by distributed [shared] leadership where the emphasis is on supporting others, team working, collaboration, removing barriers to communication, encouraging a questioning approach, promoting personal development, and showing respect for the views of others, (AlimoMetcalfe, and Bradley,2009).

The expectation is that the above will contribute to effective team processes, in particular, improved decision making and better communication between different team members and with other teams, and encourage shared learning and knowledge exchange between team members.

\section{Outputs}

The final part in the model relates to actual outputs or outcomes which may be tangible and/ or non- tangible. This depends on the combination of, and interaction between, inputs and processes, in particular, how this effects the achievement of appropriate results, or measures of effectiveness. In dentistry these may be expressed in terms of objective measures, such as clinical effectiveness, quality of care, patient safety, and extent of clinical innovation. Also, more subjectively, they may be expressed by the impact of such processes on team members' mental health, motivation, ability to contribute to overall objectives, patient satisfaction and user perceptions of the service. Ultimately, these may be used to measure overall performance of the team. 
It can be noted that this is a potentially problematic aspect of the model as the ultimate goal-overall effectiveness and its interpretation - may be subjective depending on the view of different stakeholders. Stakeholders with an interest in dentistry include professionals, patients, commissioners, and regulators, each of whom may have a differing perspective on overall effectiveness.

\section{Implications}

This paper has used the 'process' model as a framework with which to analyse key inputs, or antecedent factors, such as the characteristics of team members, organisational and contextual factors; and key process variables, such as leadership, decision-making, and task accomplishment, (Mathieu, et al,2008).

However, it is noted that there may be barriers to effective team working such as style of leadership, and also followership, (ie team members); teams need both the right leaders and the right followers in order to avoid 'toxic anti-team behaviours', (Ezziane, et al,2012). In dentistry both professional groups and individuals may find it difficult to engage in multiprofessional team working, one reason being that 'different interests, professional values and occupational cultures can militate against collaboration', (Ward, 2006, p194). There is also a potential issue with regard to the relationship between the dentist and other staff in that the former is likely to be owner of the practice and employer of staff. What may be needed is a 'culture shift' to facilitate the sharing of power, (Csikar, et al, 2009,p535).

Thus, shared leadership, as opposed to individual, top- down or vertical leadership, may be appropriate in dentistry. Leadership is an important influence on team process, ( AlimoMetcalfe, and Bradley,2009\}. Shared leadership is premised on a participative approach to decision making, one in which authority is dispersed if one characterises it in terms of the dimensions of team work mentioned earlier, ( West and Lyubovnikova, 2013). Shared leadership depends on the nature of team inputs and processes, such as the nature of the task, knowledge and skills of team members, or the extent to which team members are judged competent and empowered to take on a leadership role.

The latter may be facilitated by a leadership style that encourages team involvement in decision-making, consultation, and by displaying trust and empowerment, (Morison, and McMullan,2013). According to a large -scale study of teams in healthcare there are other aspects that depend on leadership style such as 'clear objectives, higher levels of participation, emphasis on quality and support for innovation', (Borrill, et al, 2000, p371). The latter will be relevant when the new dental contract is implemented using the dental quality and outcomes framework and care pathways approach, ( Hatton, 2017). It is argued, therefore, that: 'it is not simply what you do, but how you do it', (Alimo-Metcalfe, and Bradley,2009).

This emphasis on 'how' to do it requires nurturing and supporting, thus, 'effective teams cannot be created without appropriate education and training... [and] leaders also need to be educated to lead', (Morison, and McMullan,2013). Studies have shown that team training or development actually works, indeed, 'well designed team training increases the quality of team processes and overall performance outcomes,' (Salas, et al, 2008\}. As noted, 
developing a shared approach means developing the right culture to support it. The CQC (Care Quality Commission) supports the idea of promoting learning and innovation and 'an open and fair culture' in dentistry, (Harris, 2016).

The above may be facilitated by a combination of training methods such as coaching, mentoring, or individual counselling, the aim of which is to develop the ability to 'step back' and allow 'others to develop their critical thinking and problem-solving skills', (Le Comte and McClelland, 2017, p318). This is said to be compatible with shared leadership. In addition, group methods may be employed, such as team building and group awareness training. The overall aim is to facilitate shared learning and inculcate the right values and behaviours underpinning a shared approach. Of particular importance is the need to develop shared cognition or understanding as a basis for maintaining and improving team performance, (Salas et al 2008, p541). This may involve transformational learning involving critical reflection on experience, and contributing to the development of new interpretations or mental constructions of reality, (Brown and Posner, 2001, p274).

These developmental activities are best undertaken in a shared learning environment. The advantage of this is that it develops skills in collaboration that may help remove professional boundaries. In order to facilitate this, 'health professional students need opportunities to spend time together, to learn and to work together in meaningful ways,' (Hall, 2005 ). This is supported by the GDC who say that potential registrants should 'have the opportunity to develop in a team environment as early as possible in their training', (GDC, 2011). This is being addressed during training in the dental schools, and, as a result, the next generation of dentists, (and DCPs), should be trained in an environment which values inter-professional learning.

The overall advantages of adopting shared leadership and effective team working include: improved decision making, team motivation, shared learning, skill mix, work load balance, improvement in outcomes, reduced costs, (Ezziane, et al,2012), and benefits for patient safety, (Xyrichis, and Ream, 2008). With regard to the latter, it has been reported that half of medical errors in a US study of hospitalised patients could have been prevented by 'effective teamwork and better communication between caregivers', (Buljac- Samardzic, et al,2010). Similarly, 'the patient safety literature is littered with accounts of poor teamwork leading to adverse events', (Jeffcott, and Mackenzie, 2008). Dealing with patient safety and clinical error are high priority in the NHS and a common approach has been to apply an organisational model exploring task, team and situational factors, (Waring, McDonald, and Harrison, 2006).

\section{Conclusion}

This paper has explored team working in dentistry. It has sought to provide insights into team working using a 'process' model of team effectiveness, ( Borrill, et al,2000; Ezziane, et al,2012). It has drawn attention to the importance of key variables in the process of effective team working and the implications for dentistry. Such variables, alongside key inputs, collectively determine the likelihood of the team achieving the desired outputs. In 
particular, it is said that 'clear leadership contributes to effective team processes, [and ] to the effectiveness of performance', ( Borrill, et al,2000).

A key aspect in terms of developing effectiveness may be adopting shared as opposed to individual approach to leadership. This ensures that the full range of experience and skills within the team are utilised for the benefit of patients and the organisation.

It is concluded that team working remains important in dentistry and may be more so with changes to the organisation and delivery of dentistry, and the possibility of changing roles and responsibilities associated with initiatives like skill mix and enhanced roles of therapist/ hygienists.

\section{References}

Alimo-Metcalfe, B and Bradley, M, (2009), Cutting Crew, Health Service Journal, 7 May p 8-9

Bleakley, A (2013) Working in teams in an ear of 'liquid' healthcare: what is the use of theory? Journal of Interprofessional Care, 27: 1 pp18-26

Borrill C West, M, Shapiro, D and Rees, A, (2000) Team working and effectiveness in healthcare, British Journal of Healthcare Management, Vol 6 No 8 pp 364-371

Brown, L, M. and Posner, B, Z. ( 2001), Exploring the relationship between learning and leadership, Leadership and Organisation Development Journal, 22, 6 pp274-280

Buljac- Samardzic, M, Dekker-van Dorn, C M, van Wijngaarden, J D H, and van Wijk, K P, (2010) Interventions to improve team effectiveness: A systematic review, Health Policy, 94 pp 183-195

Csikar, J, I, Bradley, S. Williams, S, A, Godson, J, H. and Rowbotham, J, S. (2009), Dental therapy in the UK: part 4. Teamwork-is it working for dental therapists? British Dental Journal, Vol 207, 11, 12 December, pp529-536

Ezziane, Z, Maruthappu, M, Gawn, L Thompson, E A, Athanasiou T, and Warren, O J, (2012), Building effective clinical teams in healthcare, Journal of Health Organisation and Management, Vol 26, No 4, pp428-436

GDC ( 2013), a, Looking Ahead-Changes to dental provision in the UK and the implications for the General Dental Council, GDC, London

GDC, (2013), b, Standards for the dental team, GDC London GDC, (2011), Preparing for Practice: Dental team learning outcomes for registrants, GDC, London

Gopee, N. and Galloway, J, ( 2009), Leadership and Management in Healthcare, Sage, London

Hall, P, (2005), Interprofessional teamwork: professional cultures as barriers, Journal of Interprofessional Care, 19, 1. Pp188-196 
Harris, R, ( 2016), CQC-lessons from the new approach, BDJ in practice, March

Hatton, S, (2017), NHS contract reform: are you ready?, Dentistry. Co.UK, 13 February, Available at: http://www.dentistry.co.uk/2017/02/13/nhs-contract-reform-are-you-ready/

Jeffcott, S A, and Mackenzie, C F (2008), Measuring team performance in healthcare: Review of research and implications for patient safety, Journal of Critical Care, 23, pp 188-196

Le Comte L, and McClelland, B, ( 2017 ) An evaluation of a leadership development coaching and mentoring programme, Leadership in Health Services, Vol 30, 3,pp309-329

Mathieu, J, Maynard, M T, Rapp, T and Gilson, L, (2008) Team effectiveness 1997-2007: A review of recent advancements and a glimpse into the future, Journal of Management, Vol 34 no 3 June pp410-476

Morison, S and McMullan, C, (2013), Preparing for the future: challenges and opportunities for management and leadership skills, British Dental Journal, 214 E2 pp 1-5

Myers, H, L. and Myers, L, B. (2004), Its difficult being a dentist: stress and health in the general dental practitioner, British Dental Journal, 197, 24 July pp 89-93

NHS England, (2014 a) Improving dental care and oral health-a call to action, NHS England, Leeds, February

NHS England, (2014 b), Five Year Forward View, NHS England, Leeds, October

Reeson, M, G. Walker-Gleaves, C, and Jepson, N, (2013), Interactions in the dental team: understanding the theoretical complexities and practical challenges, British Dental Journal, 215

Salas, E, Cooke, N J, and Rosen, M A. (2008), On teams, teamwork, and team performance: discoveries and developments, Human Factors, Vol 50, 3 June, pp540-547

Ward, $P,(2006)$, The changing skill mix- experiences on the introduction of the dental therapist into general practice, British Dental Journal, 200, pp193-197

Waring, J. McDonald, R, and Harrison, S, (2006)Safety and Complexity: interdepartmental relationships as a threat to patient safety in the operating department, Journal of Health Organisation and Management, Vol 20, 3 pp 227-242

West, M, A, and Lyubovnikova, J ( 2013), Illusions of team working in healthcare, Journal of Health Organisation and Management, Vol 27 issue 1 pp 134-142

Wilson, N, H, F, (2017) Hard Brexit: will there be consequences for UK dentistry? Faculty Dental Journal, April Vol 8, 2

Xyrichis, A and Ream, E, (2008) Teamwork: A concept analysis, Journal of Advanced Nursing 61 (2), pp 232-241, January 
